\title{
IT Projects in Top 3 Smart Cities in India: Existing So- lutions, Challenges and Discussions for Future Scope ${ }^{1}$
}

\author{
Vidya Dhopate ${ }^{1[0000-0001-5080-5649]}$ and Vaibhav Bahel ${ }^{20000-0002-2572-8327]}$ \\ MIT World Peace University \\ School of Electronics and Communication \\ Pune, India \\ DOI: 10.29322/IJSRP.10.11.2020.p10741 \\ http://dx.doi.org/10.29322/IJSRP.10.11.2020.p10741
}

\begin{abstract}
One of the essential pillars of developing a smart city, is the presence of efficient technology-based smart solutions. This paper aims to explore the existing smart solutions (proposed as well as implemented) in Indian smart cities by conducting a case study on the smart solutions present in Top Three smart cities in India. We discuss challenges hindering development of smart cities in India, in the context of technological advancement. We study existing solutions in smart cities worldwide which may be incorporated in Indian cities. We initiate discussions regarding the development and implementation of solutions in smart cities.
\end{abstract}

This is done with the help of information provided through reports by concerned government departments and by analyzing existing scientific and economic literature.

Keywords: Smart Cities, Pune, Jaipur, Bhubaneswar

\section{Introduction}

The concept of Smart Cities gained traction with the Smarter Planet Initiative of IBM in 2008, later giving rise to Smarter Cities initiative by IBM in 2009 [1]. In the year 2015, the Government of India rolled out the 'Smart Cities Mission' with an aim to drive economic growth and improve the quality of life for people in 100 selected cities (S. Praharaj et al. [2])

Despite the lack of a forma definition for the term 'Smart City', a Smart City embeds ICT solutions at different levels: from infrastructural (by use of sensors and ICT) to governance level by using ICT to realize e-governance (E. Bigne et al. [3] ).

Of all the desirable features listed by Ministry of Urban Development (MoUD) of India which are essential to a smart city, technology will play a vital role in aiding following crucial features [4]:

- Creating walkable localities.

- Promoting a variety of transport options.

- Making governance citizen friendly and cost effective.

- Applying Smart Solutions to infrastructure and services in area-based development in order to make them better.

In an unprecedented move, MoUD declared a 'City Challenge' to rank cities based on their performance. This was done in two stages: in the first stage, 100 smart cities were shortlisted. In stage two, the shortlisted cities submit an SCP i.e. Smart City Proposal, for their planning and development vision. Thereafter, the winning cities will be ranked by various committees and boards and be expected to implement their SCPs [4].

These rankings are based on three parameters of scoring, and the parameter which interests us, in the scope of this paper is the second parameter i.e. use of ICT in resulting in change of Administrative Efficiency [4]. The top three smart cities in India are Bhubaneswar, Pune and Jaipur respectively [4].

\subsection{Case Study: Overview}

\footnotetext{
${ }^{1}$ Both authors contributed equally

This publication is licensed under Creative Commons Attribution CC BY.

http://dx.doi.org/10.29322/IJSRP.10.11.2020.p10741
} 
While there exist unique solutions across all smart cities in India implemented with the aid of IT, for the scope of our paper, we review technological solutions in top 3 smart cities in India. There also exist IT solutions available across India in every place, but these constitute a part of the Digital India Initiative - even if these solutions assist in providing IT support to citizens, these fall outside the scope of our paper. Digi Locker app, Aarogya Setu app and other such apps are few examples of such apps.

It is prudent for the reader to note that there might be an overlap in solutions across cities. An instance of this is the 'e-challan system', which is implemented in both: Bhubaneswar and Pune. However. We only describe the implementation of the same in the city of Bhubaneswar in brief. The intention behind this decision is to expand the list of unique projects covered in this paper to give the reader a more comprehensive idea of existing IT solutions. This does not, however, imply that the projects listed under each Smart City are unique to that city alone.

There are two components to Smart City Mission (SCM) in India: Pan-city component and Area Based Development (ABD) component (D. Prasad et al [5]). ABD focuses on utilizing new technologies such as smart street lights, smart meters etc while pan-city development focuses on using Command and Control Centres for unifying heterogeneous datasets. No distinction is made between the two components in the case study for ease of understanding, since we evaluate the smart city as a cohesive whole unit.

\section{Case Study: (city-wise)}

This case study aims to shed some light on the top performing smart cities of India and top performing projects under them.

\subsection{Bhubaneshwar}

Bhubaneswar is a city with a population of 8.38 lakh residents according to the latest census [6]. The MoUD published a document containing smart solutions which have been proposed in all the smart cities across India. According to this document, the city of Bhubaneswar implements the following projects:

1. Transit operations system (maintenance and tracking) [7]

BRTS (Bus Rapid Transit System) in Bhubaneswar has intelligent features approved by CRUT (Capital Region Urban Transport) which lead to smooth operation of city bus systems. The Intelligent Transport Systems are achieved through the following key components:

\section{- $\quad$ Automatic Vehicle Location System (AVLS)}

Essentially consists of a Global Positioning System (GPS) unit which is connected to a control centre to relay real-time location of the bus. This can be used for a variety of applications such as passenger tracking, monitoring vehicular speeds, estimating stop durations, traffic hold-ups and so on. AVLS can also be used for on-board passenger information systems which provide information about stops en route as well as route details.

Currently, the AVLS captures GPS data at the rate of once every 10 seconds. However, if we wish to implement the driver behaviour prediction feature, data must be captured every 1 second.

\section{- Automatic Fare Collection System}

Refers to cashless, electronic collection of bus fare using smart cards, QR codes, mobile ticketing, etc. Hand-held Electronic Ticket Vending Machines (ETVMs) can also be used for on-board ticketing.

\section{Common card (payment and operations) [8]}

Also known as CPCS, this is a proposed smart solution costing 19.19 crore rupees, and spanning over 6 to 8 months for implementation. It focuses on facilitating payment transactions for citizen services across the city. It has been proposed to be implemented through an open-loop smart card and mobile application-based payment system. 
These services primarily include:

- Payments related to buses \& other transit modes

- Parking

- Municipal services (such as property tax and other charges)

- utilities (such as electricity and water)

- $\quad$ recreational \& retail payments.

To achieve this, at least 325 machines will be installed across the city to facilitate payment services for citizens at accessible distances. The cards under this system will also be acceptable at euro, master, visa and rupay specification terminals. The broader objective is to enable citizens in accessing a unified system for all government and non-government services.

3. Area based traffic control i.e. Intelligent Traffic Control System [9]

A project costing 14.69 crores and spanning across 15 months for its implementation, the technology used for implementation is named as CoSiCoSt (Composite Signal Control Strategy) which has been developed by C-DAC (Centre for Development of Advanced Computing). Traffic Intersections in the city utilize Adaptive Traffic Signal Control System as well as pelican blinkers (safe, two-stage crossing for pedestrians using manual buttons).

This project benefits citizens by improving travel time reliability, reduces congestion, responds faster to traffic conditions and predicts traffic volumes to adjust signal timings. These signals have been installed at 43 intersections in the Bhubaneswar Smart City.

4. E-challans for traffic violations [10]

E-challan system is used to collect fines and taxes from citizens who commit vehicular offenses in Bhubaneswar. This achieves the dual-purpose of keeping a digital record of the offenders as well as maintaining transparency in the process of collecting fines. Mobile e-challan devices generate a vehicle check report (VCR) on the spot, thus alleviating the need for a manually generated VCR.

E-challan reports are generated with SARATHI and VAHAN databases. A total of 195 e-challan devices are to be provided to officers responsible for vehicular law enforcement.

5. Centralized Command and Control Centre [11]

The Centralized Command and Control Centre in Bhubaneswar is the largest such centre in India. The command centre is a multi-purpose project. Its functions include tasks like:

Traffic management, incident management, city surveillance, handling emergency services, disaster management and much more. Various external modules have been integrated into the control centre's system to provide real-time decision support. Along with an adaptive traffic signal system, the Centralised Command and Control Centre will play a crucial role in tracking buses and municipal vehicles from a single place. It also incorporates a grievance redressal system.

Apart from these, other notable projects of interest to mention are: Smart Parking System, Emergency Response, Smart City Buses, Digital Kiosk.

\subsection{Pune}

Pune is a city with a population of 31.2 lakhs residents according to the latest census [6].

The MoUD published a document containing smart solutions which have been proposed in all the smart cities across India. According to this document, the city of Pune implements the following projects: 
1. Traffic Mobile App [12]

This mobile application is launched under the name 'Pune Traffic App' by Pune Police in association with HardCastle GIS and Turnhere Designs, occupying around 9MB of memory space. Within its relatively compact size, it offers a host of features in English as well as the vernacular language Marathi, majority of which are as listed below:

- Exhaustive list of police stations in the city as well as a GPS feature to locate the police station in the city closest to the app user's current location.

- Details of police personnel and their numbers; one-tap calling feature.

- Detailed list of traffic violations and fines associated with them.

- $\quad$ RTO approved Day and Night fare charts for Auto Rickshaws; also provides information regarding prepaid auto fares from bus station and railway station.

- Detailed list of emergency contact numbers such as hospitals, ambulance services, blood bank, fire brigades, crane services.

- Push notifications for updates on traffic rules and safety tips; live traffic details available on real-time basis.

2. Smart Metering for Water [13]

One of the most widely successful smart projects which has been successfully implemented in Pune Smart City, it is an innovative project for $24 \mathrm{x} 7$ water distribution. Smart Water Meters have been installed in households in various localities across the city. Upto 40-45\% of water is lost before it reaches the end-customer in India. A company which specializes in providing smart solutions for water conservation -- Sensus iPerl -- is the start-up to which this ambitious initiative has been outsourced, claims that it will reduce this statistic to $15-20 \%$ loss of water supply.

325,000 smart water meters are to be deployed in the city over the course of next three years. These deal with two major segments: residential segment and commercial and industrial segment. These are monitored tailored to their respective behaviour sets. The smart water meter consists of three major parts:

- The meter i.e. the hardware itself

- Communication i.e. method of data transmission for the meter

- Data Analysis of communicated observations

This AI and IoT based technology can help detect customer-side leakage and pinpoint the exact location of the leak as it occurs.

3. CCTV Surveillance [14]

Pune city surveillance, referred to as PCS, is a holistic and integrated video surveillance system. It is beneficial in attaining the following four objectives:

- Support Pune police to Maintain law \& order - Consists of a 24x7 live video feed, accompanied by a variety of automatic alerts.

- Act as an aid to investigation

- Improve traffic Management - in-built video analytics are leveraged to generate automatic alerts for traffic violation. 
- Help in deterring and detecting criminal activities - Automatic alerts for crowd gathering, suspicious objects, suspicious loitering, automatic number plate recognition, aid the police to detect and deal with criminal activities efficiently.

The PCS system consists of a total of 1363 surveillance cameras, 78 of which are ANPR (Automatic Number Plate Recognition) cameras. They are spread across 412 locations throughout the city, along with 22 locations for the ANPR cameras. Live feed from 150 cameras at a time can be viewed at the Command and Control Centre in Pune.

4. Mobile app based SWM and cleanliness monitoring

SWM is an abbreviation for Solid Waste Management. Multiple applications exist to achieve the unified purpose of cleanliness monitoring and solid waste management in the city. At the forefront of this are two organizations: SWaCH (Solid Waste Collection and Handling services) and APCC (Adar Poonawalla Clean City) [15].

$\mathrm{SWaCH}$ has a website where interested citizens can contact them to join their movement, organize drives or browse information regarding initiatives by them [16]. It has partnered with Pune Municipal Corporation (PMC). It saves PMC around Rs. 15 Crore annually.

APCC App is the first of its kind movement started in Pune, with the APCC movement being a 100-crore initiative. It has around 2000 trucks and employs 400 employees. State-of-the-art machines are used to work towards these goals, in tandem with an app. The app is designed with the following features.

- Tracking location of smart garbage picking machines and bins.

- Upload an image of garbage along with details on the app; these are later processed by the server before being redirected to the nearest vehicle for clearing. On clearance, the user receives a message.

- View details of garbage details by other users as well as self-reports.

5. Intelligent road asset management [17]

The Pune Smart City Development Corporation Limited (PSCDCL) introduced a Road Asset Management System (RAMS) for better development of city roads by preparing a database of past three years. The project will require one year for data collection and succeeding three years for implementation, further data collection and data updation, and the initial target road length to be covered is around 2000 kilometres.

The operationality of the project is as follows:

- Collection of road assets and infrastructural data to assist in decision-making process, priority allocation to road servicing as well as providing data inputs so that the Municipal Corporation makes better budgeting policies.

- Launching a web-based tool for use by road engineers so as to centralize the process of road asset management.

- $\quad$ To provide accurate data analysis as judicious allocation of maintenance funds is dependent on this analysis.

- Determine the cost associated with providing various levels of serviceability for any given road section.

Apart from these, other notable projects of interest to mention are: ICT enabled billing and recovery department, grievance redressal through web, app and phone, smart bus stops, public Information system, WIFI- IT connectivity, Area based traffic control. 


\subsection{Jaipur}

Jaipur is a city with a population of 30.7 lakhs residents according to the latest census [6].

The MoUD published a document containing smart solutions which have been implemented in all the smart cities across India. According to this document, the city of Jaipur implements the following projects:

1. Transit Operations system (maintenance and tracking) [18]

JSML is going to introduce a Passenger Information System in the public transport of Jaipur. With the introduction of the Passenger Information System, the commuters would be able to know the exact schedule and location of public transport in Jaipur as well as about the seat availability in JCTSL buses and Jaipur metro. Once this system is in place the main features would include:

- Schedule of JCTSL buses and Jaipur metro

- Location of buses and metro

- Seat availability

- Details for cabs

2. Common Card (payment and operations) [19]

The project aims to provide a common platform for the following services:

- Metro

- $\mathrm{Cab}$

- $\quad$ Buses

- $\quad$ Parking

- ATM

- Retail outlets

- Restaurants

Some advantages are:

1) Increased Accountability

(b) Reduces Fraud as it introduces a common platform which can be easily monitored.

(b) Promotes Cashless Transactions and provides a platform with his-tory and incentives.

(b) Provides a platform for feedback and bags on the room for im-provement.

2) Improved Efficiency

(a) Handles complex tariff structures as it is electronically computed.

(b) Automates collection of daily fares, improving efficiency and eliminating middle men.

3) Improved Convenience

(b) Single card is introduced, connecting to all the services.

(b) No Queues for tickets which saves time and aims for a seamless operation.

(b) NFC enabled 
3. Public transit and traffic operations management centre [18]

Under this project to make Jaipur's Public Transport Smarter, a control room would also be established in the city. From this control room, the movement of each and every city bus would be tracked through the Vehicle Tracking System (VTS) in each vehicle and also the GPRS enability. The people at the control room would also be able to see who boards the bus at designated stops through CCTV.

4. GPS tracking and optimisation of routes of garbage trucks: [20]

A Vehicle tracking system was proposed to track garbage trucks, which would help in optimizing tracks and fleet for the same.

The proposal included:

- Vehicle tracking system (VTS)

- Fleet management

- People management / attendance monitoring

- Route optimization / Service scheduling

- Monitoring of Door to Door collection

- Integration of weather data

- Vehicle maintenance module

- Bin monitoring

- Multiple touch points for complaints registration \& redressal

\section{Journey Planner [18]}

The App is proposed to streamline Jaipur's public transport sector. It is proposed to have:

- the possible routes available to make the trip

- the frequency and timing of JCTSL buses_along with fare information

- the latest metro routes including information about the route, frequency and fare

\begin{tabular}{|l|l|l|l|}
\hline $\begin{array}{l}\text { Sr } \\
\text { No }\end{array}$ & City & App Project Name & Brief Description \\
\hline 1 & Bhubaneswar & $\begin{array}{l}\text { Integration of public and privately owned } \\
\text { (publicly accessible) parking availability } \\
\text { data on to common platforms and parking } \\
\text { mobile apps. }\end{array}$ & $\begin{array}{l}\text { Parking availability data on to common platform and parking } \\
\text { mobile app }\end{array}$ \\
\hline 2 & Bhubaneswar & $\begin{array}{l}\text { Emergency response intake through call, } \\
\text { app or panic-button, CAD; First Responder } \\
\text { Coordination }\end{array}$ & $\begin{array}{l}\text { Emergency Response and City Incident Management } \\
\text { through app }\end{array}$ \\
\hline 3 & Bhubaneswar & Bus Services Operations & $\begin{array}{l}\text { Passenger Information systems on-board and at bus shelters. } \\
\text {; Mobile App for Journey Planning and IEC to enhance Bus } \\
\text { Transit Usage }\end{array}$ \\
\hline 4 & Pune & $\begin{array}{l}\text { Bus System Intelligent Traffic Manage- } \\
\text { ment System (ITMS) }\end{array}$ & $\begin{array}{l}\text { Smart Public Transport for Pune - Real - time tracking of } \\
\text { buses using GPS - Maintenance using vehicle health moni- } \\
\text { toring system - Passenger information system (PIS) with }\end{array}$ \\
\hline
\end{tabular}




\begin{tabular}{|l|l|l|l|}
\hline 5 & Pune & e-Challan & LED screens in buses and bus -stops \\
\hline 6 & Pune & $\begin{array}{l}\text { Mobile App and Website for Pan City Wa- } \\
\text { ter }\end{array}$ & $\begin{array}{l}\text { G - challan systems with smart challan payments Cops to be } \\
\text { equipped with android based e-challan tablets } \\
\text { mobile app }\end{array}$ \\
\hline 7 & Jaipur & Air Quality Monitoring with App & $\begin{array}{l}\text { Air Quality Monitoring Application to be developed to create } \\
\text { mega data and to be accessible by citizens }\end{array}$ \\
\hline 8 & Jaipur & $\begin{array}{l}\text { Mobile application enabling citizens to } \\
\text { report street problems connecting to e- } \\
\text { governance portal }\end{array}$ & $\begin{array}{l}\text { Development of 'In My Street App enabling citizens to report } \\
\text { street problems }\end{array}$ \\
\hline 9 & Jaipur & $\begin{array}{l}\text { Incident alerts app, Helpline and SOS Mo- } \\
\text { bile number and Panic Buttons }\end{array}$ & $\begin{array}{l}\text { Development of app for Safety of citizens especially chil- } \\
\text { dren, woman and elderly }\end{array}$ \\
\hline 10 & Jaipur & $\begin{array}{l}\text { Journey Planner App } \\
\text { wevelopment of app for streamlining Jaipur's Public } \\
\text { Transport sector. This app would integrate all means of the } \\
\text { city's public transport sector which includes JCTSL buses, } \\
\text { Jaipur metro and cab services. }\end{array}$ \\
\hline
\end{tabular}

Table (1): App-Based Solutions in Smart Cities

\section{Challenges}

A plethora of challenges are experienced in the Smart City Mission, and existing literature points towards a variety of factors related to governance, infrastructure, economic policies and environmental policies. A few challenges in relation to the technological context are elucidated below.

According to technological barriers to development of smart cities in the Indian context (N.P Rana, et al [21]), the following points have been brought to attention:

1. Lack of technological knowledge among the planners.

2. Lack of access to technology.

3. Privacy and security issues.

4. System failure issues due to vulnerability to cyber-attacks and other incidents such as industrial espionage, terrorism, equipment failure.

5. Integration and convergence issues across IT networks.

6. Poor data availability and scalability.

Lack of timely updates on government portals and websites makes it difficult for citizens to ascertain how far along the project is in its implementational journey - this can only be remedied by responsible governance which is intent on providing transparent and updated information for further scrutiny.

Previous policy failure in India brought about a shift in its urban policies from being infrastructure-centric to market-centric. Considering this shift in the national urban policies, it is argued that smartness in India is a vague concept. It was simplified as either the use of technology in governance and planning or as greenfield construction of urban hubs to draw international investors (D. Prasad et al [5]) which leads to underwhelming focus on other aspects of smart cities.

Focus on economic growth in smart cities in India often comes with an increase in urban inequality. (D. Prasad, et al [5]). This goes against the principle that technology in smart cities should alleviate rather than assists in maintenance of status quo in smart cities.

The smart cities mission guideline published by the Government of India does not have a clear framework or outline regarding 
how different initiatives will be coordinated for the common good of the city (S. Praharaj, et al [2]), which will act as a hindrance in IT integration, where well-defined architecture and planned conglomeration is key.

\section{Discussions}

Before beginning the discussions surrounding smart cities in India, it would do well to recollect that the Indian Smart Cities Mission is a city-driven exercise, managed by MoUD. There is a vast service delivery infrastructure gap that India needs to cover compared to countries with successful implementation of Smart Cities.

That being said, we observe a lack of artificial intelligence-based solutions in smart cities in India, despite the vast potential of the field. The successful application of AI requires a good understanding of the relationships between AI and data as well as transportation system characteristics and variables (Nikitas, et al [22]). The range of AI-based technologies which can be exploited to make a smart city technological sound in a study (Nikitas, et al [22]) is versatile and varied. Indian Smart Cities fall remarkably short of successfully implementing these.

Smart City Hackathons are conducted across major smart cities in India where youth present innovative ideas and are provided incubation for implementation of their solutions; a majority of these ideas are in the fields of IoT using drones for surveillance or using AI for computer vision or decision-making and prediction. Have these innovations been taken into consideration for smart cities at an implementational level?

From the analysis of their vision statements, it is clear that half of the top 20 smart cities overlook sub-dimensions of health, literacy, youth, as well as the vulnerable section of the society (women, differently-abled citizens, seniors and children). This is a concerning oversight (D. Prasad, et al [5]). Only five of the smart cities have proposed to improve their market through the techindustry using smart policy making. What measures should be taken to rectify this oversight?

It is evident that the smart environment dimension is not the core in any of the SCPs prioritised for smart city implementation in India (D. Prasad et al [5]). A striking contrast to this is the city of Oslo in Norway which features 3rd on global rankings of lists of smart cities. Its effort to address climate change and take concrete measures against it are a part of the reason, with aim to cut as much as $95 \%$ of its carbon emissions by 2030 . With climate change set to adversely affect India, it is necessary for Indian Smart Cities to take prudent measures to reduce its effects. Infrastructural and Technical limitations are key to lack of climate resilience in Indian Smart Cities.

Lasty, while surveillance in Smart Cities in India is still in its infancy, there is a lack of clear ethical guidelines in the CCTV surveillance mechanisms and other data-privacy surveillance mechanisms employed. Cities must figure out a solution to survey citizens while also avoiding infringement and invasion of their data privacy. This can lead to a moral dilemma and, left unresolved, lead these smart cities to experience the hazards of a 'surveillance culture' (Lyon et al [23]).

\section{Conclusion}

We have explored IT solutions either proposed or implemented in the top 3 Smart Cities in India i.e. Bhubaneshwar, Pune and Jaipur respectively. These solutions are unique to the needs of the citizens of each specific city. The paper details some of the interesting projects in the field of IT existing in these cities and elaborates on their implementation details.

While powerful IT solutions are being implemented all across India as a part of the Smart Cities Mission, much progress is left to be desired, especially on conducting a comparative analysis globally. We explore shortcomings of Indian Smart Cities and delve into the causes leading up to it. We cover the key reasons existing in current research literature which leads to technological limitations in Indian Smart Cities.

We also open a variety of key discussions regarding how smart cities in India must proceed. These are explored from a technological lens. We discuss the projects Indian smart cities can draw inspiration from and emulsify. We also look at a wide collection of sectors which can benefit from IT but which have been overlooked in the vision for Indian Smart Cities.

We hope this paper can act as a balanced reference for individuals and teams embarking upon projects related to the IT field to aid in the development of Indian Smart Cities. We make a sincere effort to present in-depth the technological angle of this issue, so that it can assist policymakers and economists exploring this issue. 


\section{References}

[ "IBM Smarter Planet," [Online]. Available: https://www.ibm.com/smarterplanet/us/en/. 1]

[ J. H. H. a. S. H. Sarbeswar Praharaj, "Urban innovation through policy integration: Critical perspectives from 100 smart 2] cities mission in India," City, Culture and Society, vol. 12, 2018.

[ C. R. R. C.-P. a. J. M. Enrique Bigne, "The Effects of eWOM in Social Media on Tourism," in 3rd International 3] Conference on Tourism Research (ICTR 2020), Valencia, 2020.

[ "smart cities, gov of india," [Online]. Available: http://smartcities.gov.in/content/. 4]

[ T. A. Deepti Prasad, "What makes Indian Cities Smart? - A Policy Analysis of Smart Cities Mission. Telematics and 5] Informatics," Telematics and Informatics, vol. 55, 2020.

[ "census," [Online]. Available: https://censusindia.gov.in/towns/town.aspx.

6]
[ "Urban
industrial,"
[Online].
Available:
https://www.urban-industrial.in/hrdpmp/igep-

7] uid/content/e15/e1536/e5085/e5146/e5148/CRUT_2.pdf.

[ "smart city bhubneswar," [Online]. Available: https://www.smartcitybhubaneswar.gov.in/common-payment-card-system. 8]

[ "smart city bhubneswar," [Online]. Available: https://www.smartcitybhubaneswar.gov.in/traffic-management-system. 9]

[ "e-challan," [Online]. Available: https://odishabytes.com/e-challan-to-collect-motor-vehicle-taxes-fines/.

10]

[ "odishatv," [Online]. Available: https://odishatv.in/odisha-news/bhubaneswar-gets-smart-command-control-centre11] 307347.

[ "pune traffic app," [Online]. Available: https://pune-traffic-app.soft112.com/.

12]

"smart metering," [Online]. Available: https://energy.economictimes.indiatimes.com/news/renewable/we-expect13] improved-momentum-in-smart-water-metering-in-india-going-forward-sensus/77805008.

[ "pmc," [Online]. Available: https://www.pmc.gov.in/en/closed-circuit-television-system-cctv.

14]

[ "aadar poonawala clean," [Online]. Available: http://www.adarpcleancity.com/.

15]

"swach," [Online]. Available: https://swachcoop.com/.

16]

"pmc," [Online]. Available: https://www.pmc.gov.in/en/road-asset-management-system-rams.

17]

[ "travel jaipur," [Online]. Available: http://cityofjaipur.com/jaipur-travel-and-transportation/getting-around-jaipur/public18] transport/public-transport-in-jaipur-to-get-smart-smart-city-project/.

[ "scribd," [Online]. Available: https://www.scribd.com/presentation/410107484/Jaipur-Smart-City-Common-Mobility19] Card-RL.

[ "smart city jaipur," [Online]. Available: http://smartcities.gov.in/upload/uploadfiles/files/Jaipur_smartcity.pdf.

20]

S. L. S. K. M. R. I. S. R. \&. Y. K. D. Nripendra P. Rana, "Barriers to the Development of Smart Cities in Indian Context," 21] Information Systems Frontiers, 2018.

[ K. M. ,. T. N. a. D. K. Alexandros Nikitas, "Artificial Intelligence, Transport and the Smart City: Definitions and 22] Dimensions of a New Mobility Era," sustainability, 2020.

[ D. Lyon, "Digital Citizenship and Surveillance| Surveillance Culture: Engagement, Exposure, and Ethics in Digital

23] Modernity," International Journal of Communication, vol. 11, 2017. 